\title{
A meta-analysis of the added value of diffusion weighted imaging in combination with contrast-enhanced magnetic resonance imaging for the diagnosis of small hepatocellular carcinoma lesser or equal to $2 \mathrm{~cm}$
}

\author{
HAILONG LAN ${ }^{1,2}$, GUISEN LIN ${ }^{3}$ and WEIZHI ZHONG ${ }^{1}$ \\ ${ }^{1}$ Department of Radiology, Wuchuan People's Hospital, Wuchuan, Guangdong 524500; ${ }^{2}$ Department of Radiology, \\ Xiaolan Hospital Affiliated to Southern Medical University, Zhongshan, Guangdong 528000; ${ }^{3}$ Department of Radiology, \\ Second Affiliated Hospital of Shantou University Medical College, Shantou, Guangdong 515000, P.R. China
}

Received October 23, 2019; Accepted June 2, 2020

DOI: 10.3892/ol.2020.11805

\begin{abstract}
Diffusion weighted imaging (DWI) has been found to increase the sensitivity in the diagnosis of small hepatocellular carcinoma (HCC), although additional studies are required to confirm its value. The aim of the present study was to explore the diagnostic performance of DWI combined with contrast-enhanced magnetic resonance imaging (MRI) for small HCC by performing a meta-analysis. Literature databases (PubMed, Embase, Web of Science and Cochrane Library databases) were searched to identify studies reporting the sensitivity and specificity of MRI with DWI for the diagnosis of small HCCs. Pooled sensitivity and specificity were generated using a bivariate random effect model. Multilevel mixed-effects logistic regression analysis was used to examine the value of DWI combined with conventional MRI. A total of 837 small HCCs and 545 benign liver lesions from 10 studies were included. The overall sensitivity and specificity of DWI combined with contrast-enhanced MRI was 0.88 (95\% CI, 0.80-0.93) and 0.90 (95\% CI, 0.81-0.95), respectively. Compared with that in contrast-enhanced MRI, DWI with contrast-enhanced MRI had a significantly higher sensitivity for the diagnosis of small HCC $(\mathrm{P}=0.01)$ while there was no significant difference in the specificity $(\mathrm{P}=0.603)$. The present meta-analysis suggests that DWI combined with contrast-enhanced MRI may increase the sensitivity, whilst maintaining high specificity for the diagnosis of small HCCs with a diameter $\leq 2 \mathrm{~cm}$.
\end{abstract}

Correspondence to: Dr Hailong Lan, Department of Radiology, Wuchuan People's Hospital, 12 Jiefang North Road, Wuchuan, Guangdong 524500, P.R. China

E-mail: lanhailong_1988@163.com

Key words: small hepatocellular carcinoma, diffusion weighted imaging, contrast-enhanced magnetic resonance imaging, meta-analysis

\section{Introduction}

The incidence of hepatocellular carcinoma (HCC), which predominantly occurs in patients with a cirrhotic liver, is increasing and has become the second leading cause of cancer-associated death worldwide, accounting for 746,000 cases or $9.1 \%$ of all cancer death in 2012 (1). Amongst all the potential treatment options, including local reginal therapy, resection and chemotherapy, liver transplant has the most favorable outcome and results in improved overall survival times (2). The United Network for Organ Sharing and the Organ Procurement and Transplantation Network have increased the priority allocation of liver transplants for patients with HCC nodules between 1-2 cm (3). In addition, resection of very early stage HCCs, with a diameter $<2 \mathrm{~cm}$, increases the overall 5-year survival rate of patients (4). Therefore, diagnosing $\mathrm{HCC}$ at an earlier stage, particularly for small HCC lesions is important. Currently, dynamic contrast enhanced magnetic resonance imaging (MRI) is the most accurate imaging modality for the diagnosis of HCC (5). Notably, up to $45 \%$ of small HCC cases may be misdiagnosed, according to the MR diagnostic criteria (6). Diffusion weighted imaging (DWI) MRI has been found to increase the sensitivity in the diagnosis of small HCC when combined with conventional MRI (7-10). However, all of these previous studies were individual studies. Differences in population characteristics, patient risk estimation, study design and imaging protocols reduce the reliability of the results from individual studies; therefore, additional studies are required to confirm its value. Thus, the present meta-analysis was performed to determine the value of DWI combined with contrast-enhanced MRI for small $\mathrm{HCC}$, with diameters $\leq 2 \mathrm{~cm}$.

\section{Materials and methods}

Literature search. A systematic literature search using PubMed (https://pubmed.ncbi.nlm.nih.gov), Embase (https://www. embase.com), Web of Science (https://apps.webofknowledge.com) and Cochrane Library databases (https://www. 
cochranelibrary.com) were performed independently by three radiologists to identify articles published prior to June 2019, using the key words 'hepatocellular carcinoma', 'liver cancer', 'liver cell carcinoma', 'magnetic resonance imaging', 'diffusion magnetic resonance imaging', 'diffusion MRI' and 'diffusion weighted MRI'. Related citations in eligible articles were also assessed for inclusion.

Study selection. The three radiologists reviewed all 2447 abstracts after duplication removal and subsequently the full text of the 119 articles was obtained if the following inclusion criteria was fulfilled: i) Included the diagnostic accuracy of MRI with DWI for HCC; ii) constituted original research rather than a meta-analysis, a review article, case report or case series; iii) published in English; and iv) results are from humans and not animals. For the studies in which the full text was reviewed, the following inclusion criteria was used: i) Included original data regarding the detection of small HCC lesions, $\leq 2 \mathrm{~cm}$; ii) included both contrast-enhanced MRI and DWI; iii) included sufficient data, with $>20$ patients to calculate true positive (TP), false positive (FP), false negative (FN) and true negative (TN) for constructing a $2 \times 2$ contingency table; and iv) patients diagnosed with hepatic lesion using pathological analysis (surgical resection, explant and/or biopsy) or imaging from follow-up according to the guidelines for standardization of liver imaging, diagnosis, classification and reporting of hepatocellular carcinoma (3). In addition, articles from the same institution, which included an overlap period of patient recruitment were considered to have an overlapping population. In these cases, the study, which had the larger number of small HCCs cases, was included. If there were disagreements between the three investigators, the consensus amongst the three radiologists was used to resolve the disagreement. Disagreements were resolved following discussions between the three investigators, until at least two of the investigators reached the same conclusion. Attempts were made to contact the authors of the article only if data for the $2 \times 2$ contingency table was not fulfilled from the inclusion criteria (authors of two articles had been contacted for this study). A total of 109 studies were excluded according to the following exclusion criteria: i) They were not relevant to the present meta-analysis if they fit one of the followings conditions: Cancer type includes malignant cancer other than HCC, such as cholangiocarcinoma, hepatoepidermoid carcinoma and metastatic cancer; diagnosis of HCC using a combination of multiple imaging modalities; size of $\mathrm{HCC}$ lesions $>2 \mathrm{~cm}$; ii) the size of the HCCs was not specified; iii) they evaluated previously treated HCCs; iv) the specificity was not evaluated; v) there was a lack of sufficient data to construct a $2 \times 2$ contingency table; and vi) there was study population overlap. A total of 10 studies were included for analysis. In addition, the reference list of these 10 studies was reviewed. Once any of the studies fulfilled the inclusion criteria but not the aforementioned exclusion, they were not included for analysis. No studies were excluded in the process.

Data extraction and quality assessment. A total of two investigators reviewed the included studies and extracted the relevant details for the meta-analysis. The study characteristics extracted included the authors of the study, year of publication, country of origin, number of overall patients, overall size of HCC, cause of liver cirrhosis, study design (prospective or retrospective image interpretation), study period, b value of DWI, MRI field strength, number of HCCs which were $\leq 2 \mathrm{~cm}$, number of benign lesions, type of benign hepatic lesions, reference standard and number of readers. The number of readers is important as diagnosing HCC lesions by multiple readers or radiologists increases the accuracy of the diagnosis, which improves the reliability of the studies.

Data for the diagnostic value of DWI combined with contrast-enhanced MRI for small HCC lesions were extracted to construct a $2 \times 2$ contingency table. If the sensitivity and specificity were reported by multiple radiologists, the average sensitivity and specificity scores were reported to avoid underor overestimation of the diagnostic accuracy, which occurred in 1 out of 10 of the included studies. In addition, raw data for the diagnostic value of contrast-enhanced MRI was extracted if available for the construction of a $2 \times 2$ contingency table, which was available in 8 out of the 10 included studies. For studies with DWI, the information of whether a preset apparent diffusion coefficient (ADC) cutoff value to diagnose HCC was also extracted for analysis, which was available in 1 out of 10 included studies). All the data were analyzed using Stata version 14.0 (https://www.stata.com).

The quality of the included studies was assessed using the Quality Assessment of Diagnostic Accuracy Studies (QUADAS-2) (11). Exclusions of patients with lesion smaller $(<1 \mathrm{~cm})$ was considered inappropriate since this increased the selection bias. Any reference standard to diagnose small hepatic lesions $(\leq 2 \mathrm{~cm}$ ), other than pathological analysis (biopsy, surgical resection and explant), for example, imaging follow-up, was considered unlikely to lead to correct classification of the target condition and may introduce bias. Diagnosis using reference standard based on imaging follow-up or subsequent transcatheter arterial chemoembolization were considered to include the knowledge of the results of the index test. In addition, the risk of bias for reference standard results based on biopsy or resection were considered unclear due to the lack of information provided to the pathologist at the time of assessment. An interval of $>90$ days between MRI scan and reference standard examination was considered inappropriate since during such a long interval, new tumorous growth adjacent to the targeted mass identified by MRI scan or reference standard examination may happen; the targeted mass may become larger over $2 \mathrm{~cm}$; patients may receive treatment that may change the size and the cell composition of the targeted mass. All the aforementioned criteria were used by the two evaluators to specify the QUADAS-2, which was generally developed for quality assessment for all meta-analysis, to assess the quality of the included studies for the present analysis.

Statistical analysis. All the data were analyzed using Stata software (version 14.0; College Station, TX, USA). The sensitivity, specificity and $95 \%$ confidence intervals (CIs) for the diagnosis of small HCC lesions using contrast-enhanced MRI with DWI were calculated using the bivariate random effects model (12), which were demonstrated via forest plots. The assessment for the sensitivity and specificity was performed on a per-lesion basis for all the included studies. The summary receiver operating characteristic curves (SROCs) were 


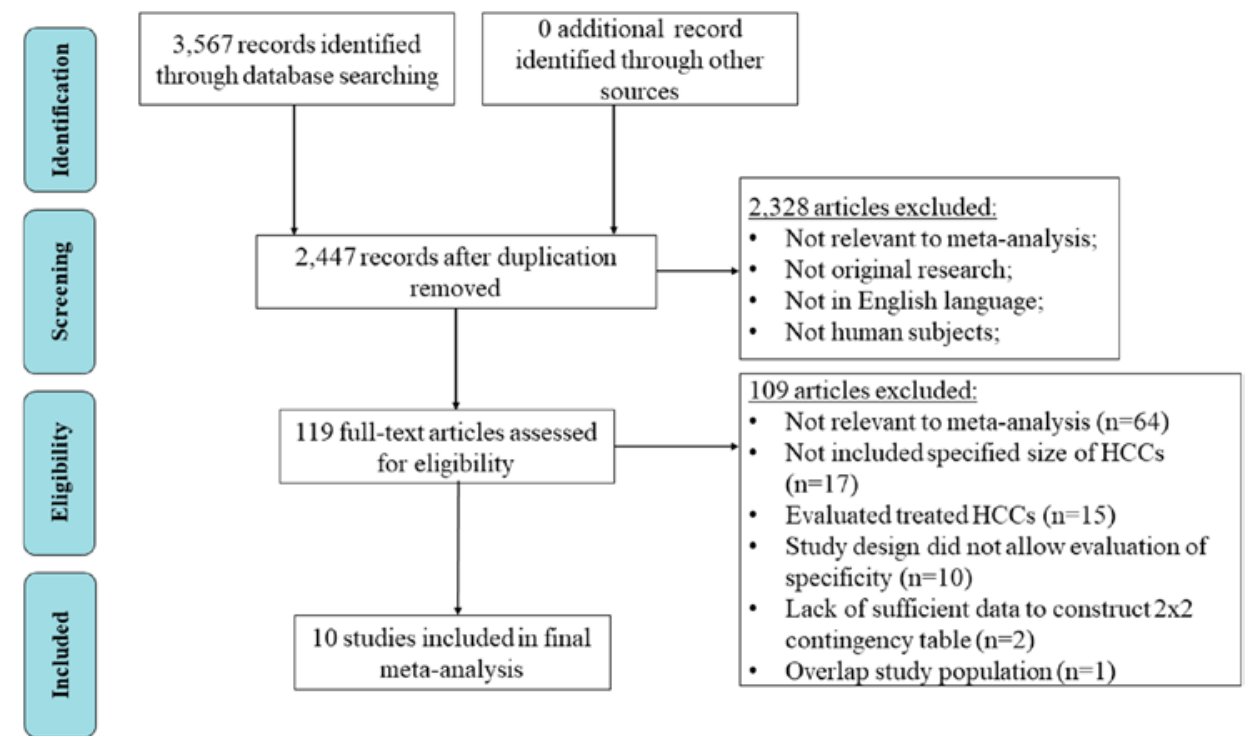

Figure 1. Flow chart for the identification of the articles included in the meta-analysis. HCC, hepatocellular carcinoma.

constructed and the area under the SROCs (AUC) of the conventional MRI with DWI and conventional MRI alone were calculated to determine the diagnostic performances. $\chi^{2}$ test $\left(\mathrm{P}<0.05\right.$ indicating significant heterogeneity) and $\mathrm{I}^{2}$ was used to determine the heterogeneity. A random effects model was used if $\mathrm{I}^{2}>50 \%$; otherwise, a fixed effects model was used. Univariate meta-regression analysis was performed according to MRI field strength (1.5 T vs. $3.0 \mathrm{~T}$ ), country of origin (Asia vs. non-Asia), study design (prospective vs. retrospective) and whether hepatobiliary phase was used in the diagnosis of small HCC. In Asia countries, Hepatitis B virus infection is the leading cause of cirrhosis which results in HCC (13). In non-Asia country, the etiology of HCC varies (13). The difference in the etiology of HCC may be the cause of the heterogeneity. This was the reason that country of origin was divided into Asia vs. non-Asia in the present study to assess the potential cause of heterogeneity. In addition, the diagnostic value of DWI combined with contrast-enhanced MRI was assessed by comparing the diagnostic performance of DWI with contrast-enhanced MRI to contrast-enhanced MRI only. The calculation of TP, FP, TN and FN for the contrast-enhanced MRI was only available for 8 of the 10 included studies $(7,8,14-19)$. Multilevel mixed-effects logistic regression analysis was used to compare the summary paired sensitivity/specificity data with a significance level of $\mathrm{P}<0.05$. Publication bias was assessed using a Deeks' funnel plot.

\section{Results}

Study selection. A flow chart following the Preferred Reporting Items for Systematic Reviews and Meta-analysis principles was used to demonstrate the selection procedure (Fig. 1). A total of 3,567 articles were initially identified. There was a total of 2,447 articles remaining following the removal of duplicates and a further 2,328 articles were excluded, following screening of the abstract. Amongst the remaining 119 studies, a total of 10 studies were included in the meta-analysis using the inclusion criteria $(7,8,14-21)$.
Summary of included studies. The summarized characteristics and the diagnostic performance of DWI combined with contrast-enhanced MRI for the included 10 studies are shown in Tables I and II, respectively. A total of 837 small HCCs with a diameter $\leq 2 \mathrm{~cm}$ and 545 benign liver lesions, with a diameter $\leq 2 \mathrm{~cm}$ was included in the meta-analysis. The TP, FP, FN and $\mathrm{TN}$ were all calculated on a per-lesion basis. Of the included studies, 6 originated from Asia, 3 from Europe and one from Egypt. In addition, seven of the studies were retrospective, and three were prospective. The reference standard for the diagnosis of HCC included pathological analysis (surgical resection, explant and/or biopsy) and imaging from follow-up. MR imaging field strength was all $\geq 1.5 \mathrm{~T}$.

Quality assessment and publication bias. Fig. 2 demonstrates the overall evaluation for the quality of the included studies using QUADAS-2. The quality of the index test was high (90\%, 9/10 studies); however, patient selection had a low score (70\%, 7/10 studies), which could be due to a lack of avoidance of a case-control design or the inappropriate exclusions during patient selection. This also increased concerns regarding the applicability of patient selection. For all the 10 included studies, some of them used pathological finding as the only reference standard to diagnose HCC. For the others, imaging follow up was used as a reference standard for patients when pathology analysis was not available. A low score was found for the reference standard ( $60 \%, 6$ of 10 studies) due to a lack of using pathological analysis as a reference standard. However, these studies used imaging follow-up as one of the reference standards for those without pathological confirmation, which has been shown to be effective for the diagnosis of HCC (22). Therefore, the concerns of bias for applicability of reference standards was low for studies using imaging follow up as one of the reference standards for patients when pathology was not used. The risk of bias for flow and timing was high for 1 study since the interval between MRI scan and the pathological analysis exceeded 90 days for some of the patients, and was unclear for 2 studies for the lack of information regarding the 


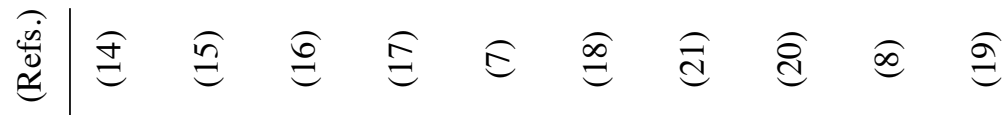

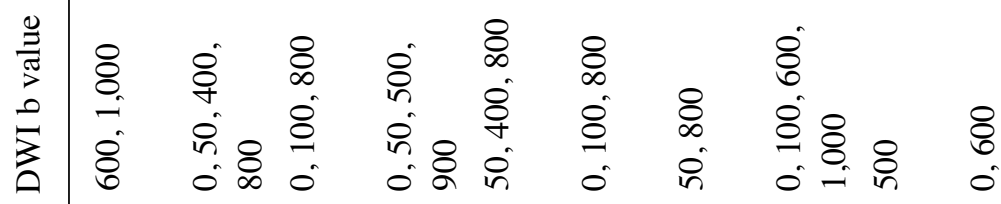

焉

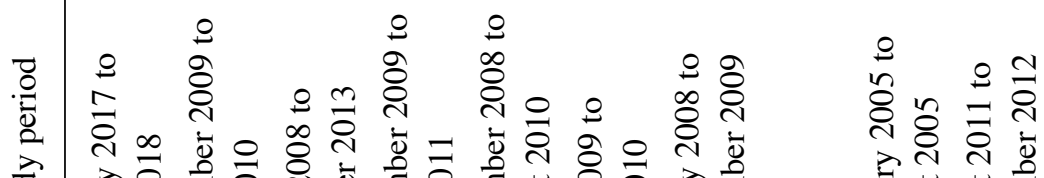

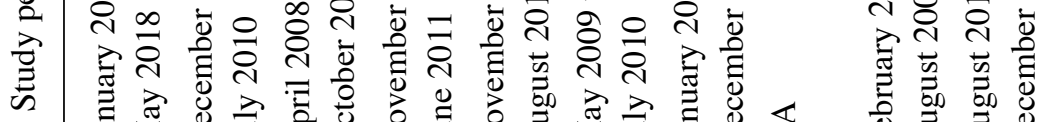

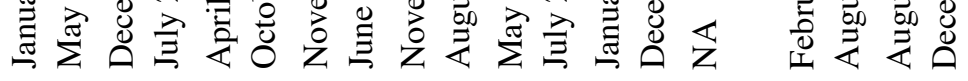

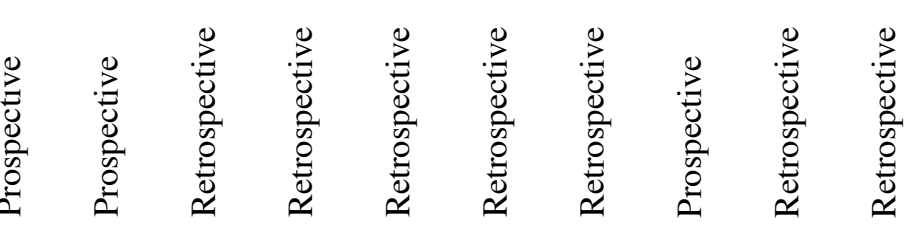

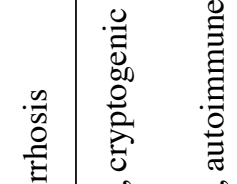

茞

$\frac{\substack{0 \\ \frac{0}{0}}}{\frac{0}{0}}$

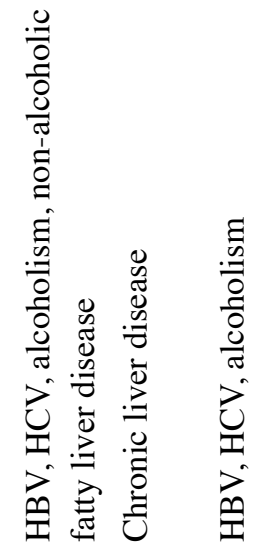

$\frac{n}{0.0}$

离党

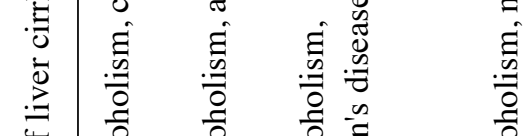

पे

政

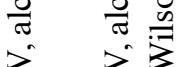

它

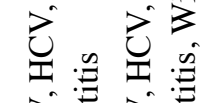

总芯畐芯芯

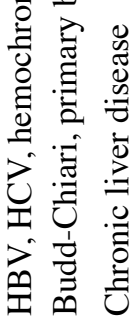

:

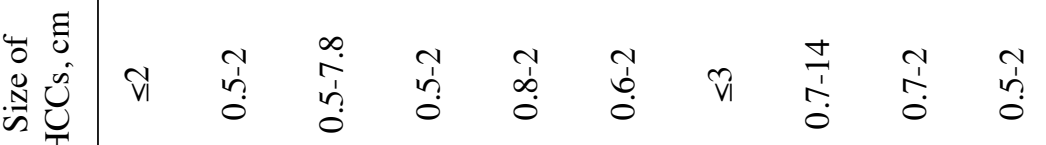

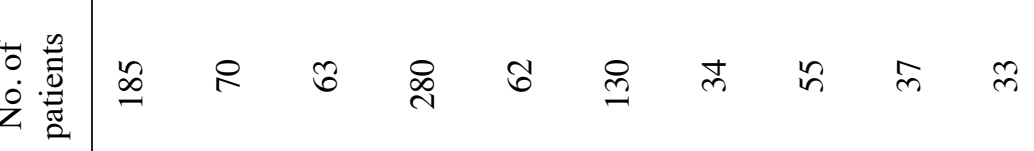

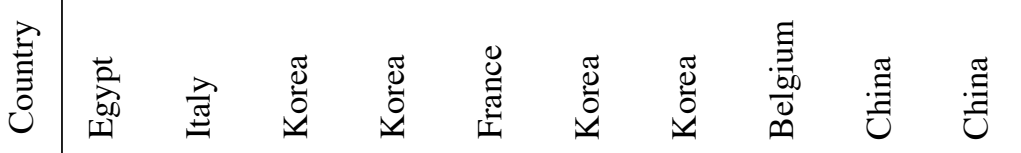

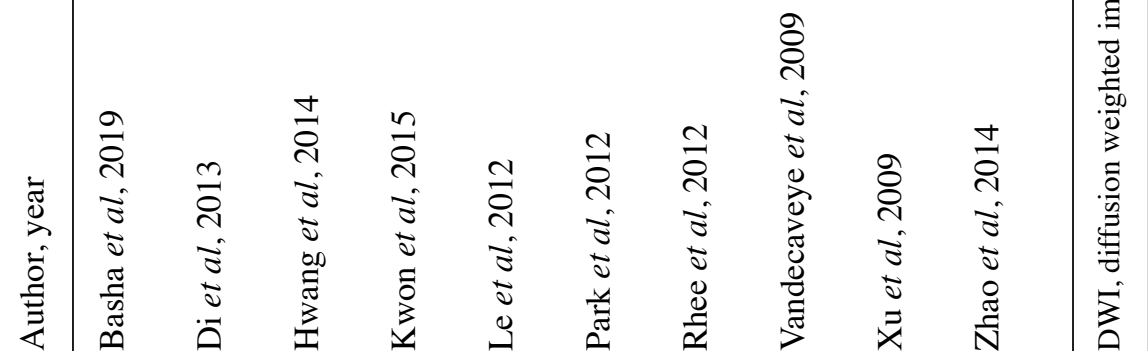




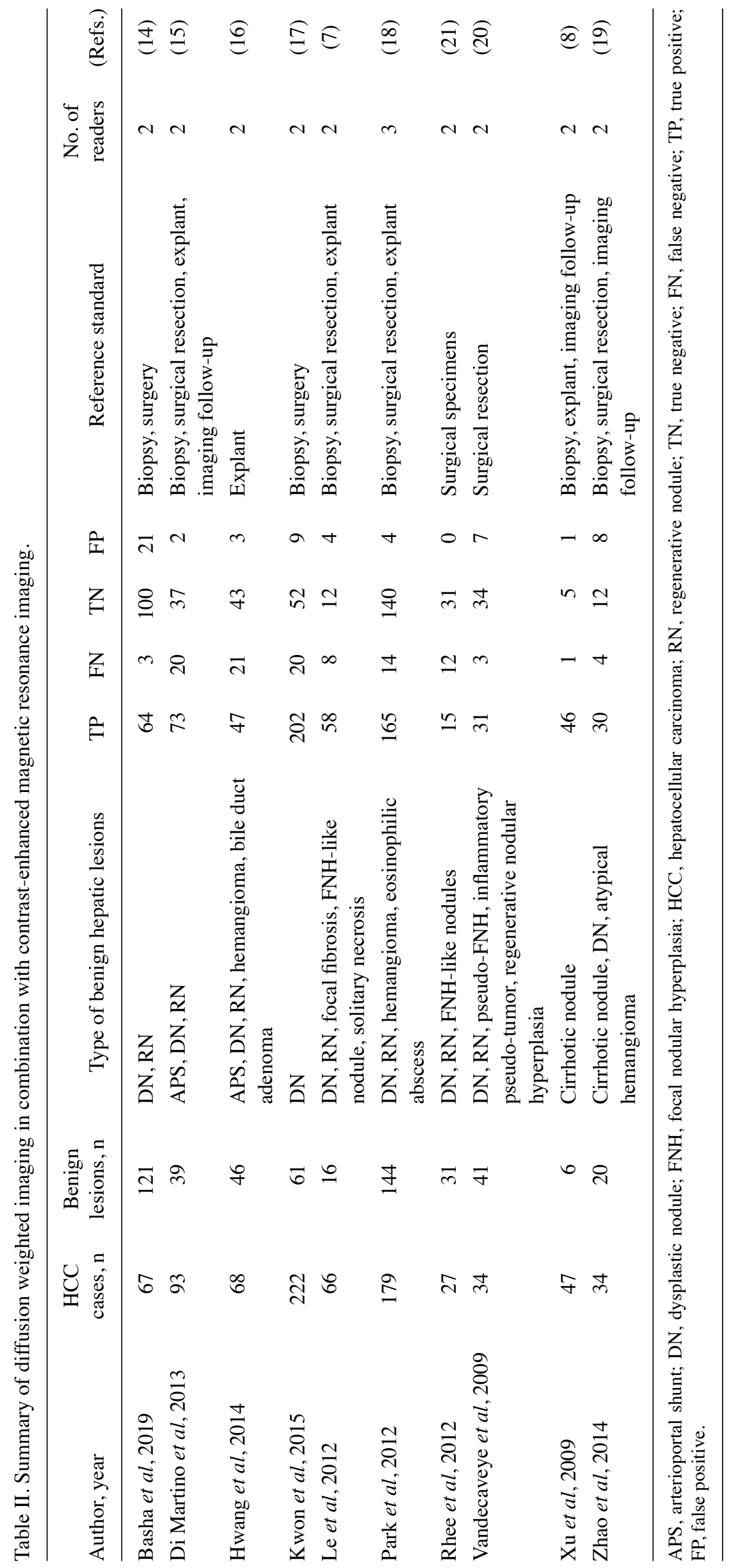



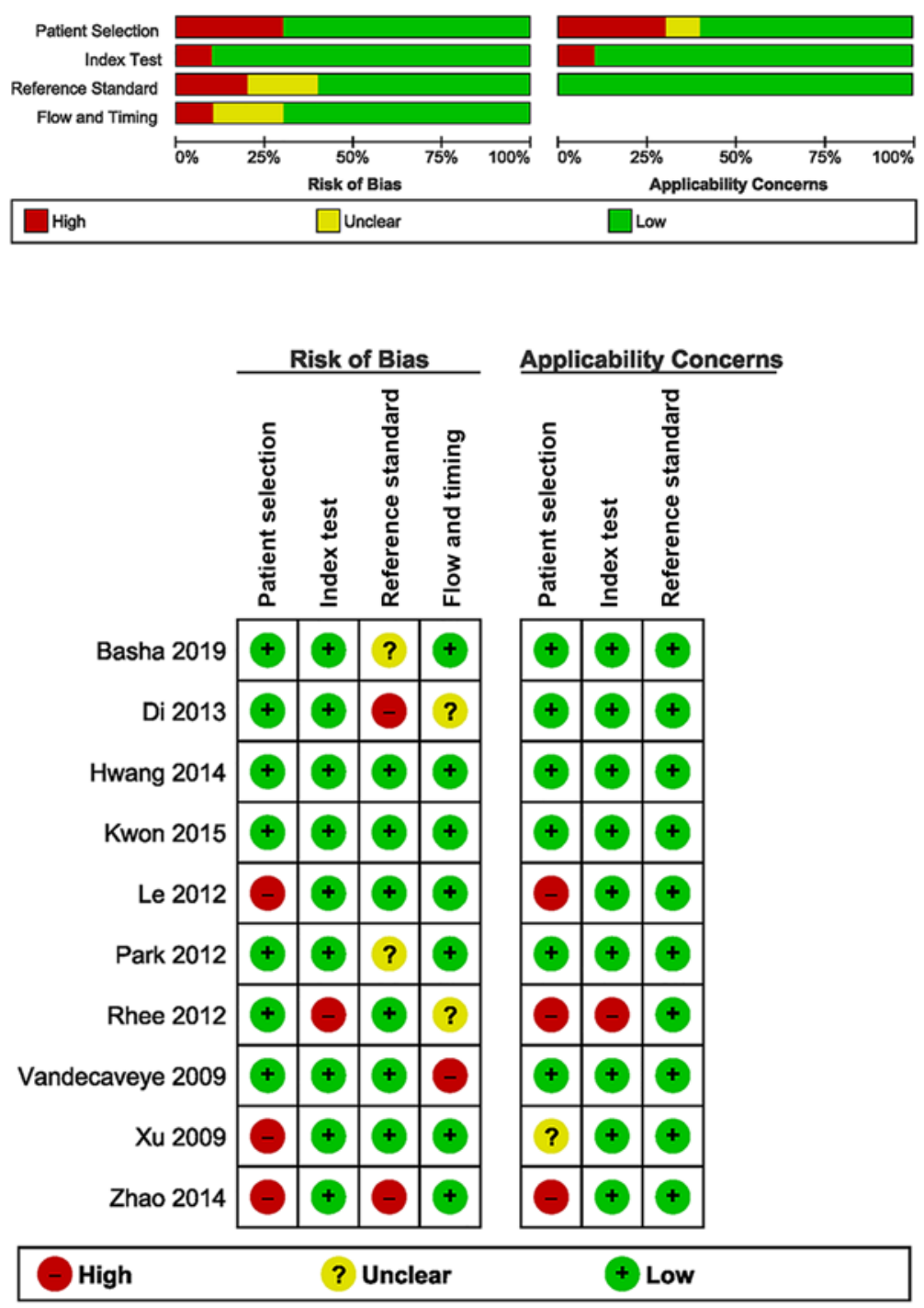

Figure 2. Quality assessment of the included studies using the Quality Assessment of Diagnostic Accuracy Studies. The red bar indicates high risk of bias; the yellow bar indicates unclear risk of bias; and the green bar indicates low risk of bias. In the lower part, details of quality assessment were shown. Green circle with ' + ' indicates low risk of bias or low concern for applicability; yellow circle with '?' indicates unclear risk of bias or unclear concern for applicability; red circle with '-' indicates high risk of bias or low concern for applicability.

time interval between MRI scan and the reference standard. The Deeks' funnel plot (Fig. 3) suggested that there was no significant publication bias $(\mathrm{P}>0.05)$.

Heterogeneity between studies. The 10 included studies demonstrated significant heterogeneity with $\mathrm{P}<0.001$ using $\chi^{2}$ test. The heterogeneity for the sensitivity ( $\mathrm{I}^{2}$ of 85.7$)$ was higher compared with that for specificity ( $\mathrm{I}^{2}$ of 78.11). In addition, there was no threshold effect found (correlation, -0.65 ; proportion of heterogeneity due to threshold effect, 0.42 ).

Synthesis of general diagnostic parameters. Fig. 4 demonstrates the forest plots of sensitivity and specificity for DWI combined with conventional MRI for the diagnosis of small HCC lesions, with a diameter $\leq 2 \mathrm{~cm}$. The pooled sensitivity and specificity were 0.88 (95\% CI, 0.80-0.93) and 0.90 (95\% CI, 0.81-0.95), respectively. The positive and negative likelihood ratio was 8.4 (95\% CI, 4.6-15.3) and 0.13 (95\% CI, 0.08-0.22), respectively. Fig. 5 shows the summary ROC curve with an AUC of 0.95 .

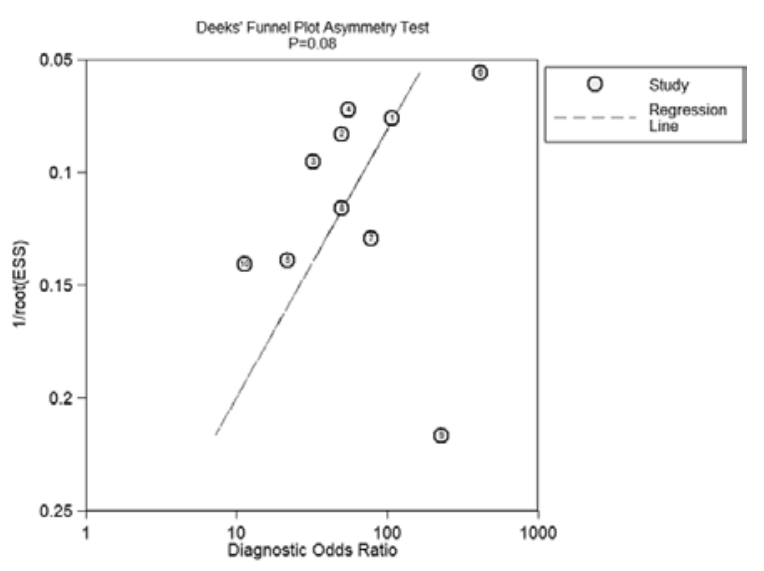

Figure 3. Deeks' funnel plot for assessment of publication bias. Potential publication exists if the calculated $\mathrm{P}<0.05 .1 / \operatorname{root}(\mathrm{ESS}): 1 / \sqrt{\text { error sun of square }}$.

Subgroup analysis and meta-regression. The results of the univariate meta-regression analysis are shown in Table III. 

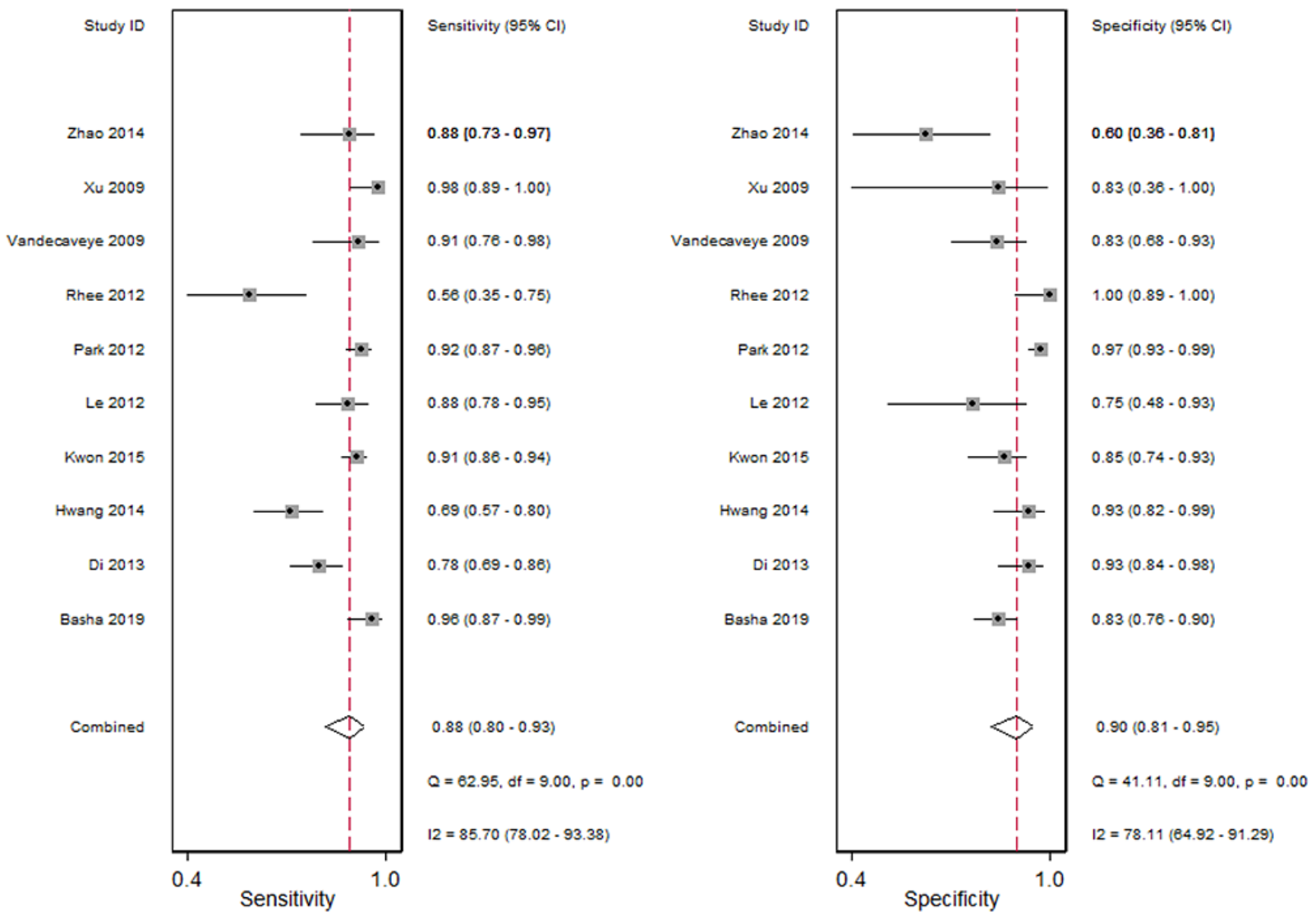

Figure 4. Forest plot of the pooled sensitivity and specificity for the included studies. Black solid horizontal lines indicate $95 \%$ CIs of each individual studies. Red dashed line indicates the pooled sensitivity or specificity for all 10 studies. The grey boxes with central black dots indicate the sensitivity or specificity for each individual study.

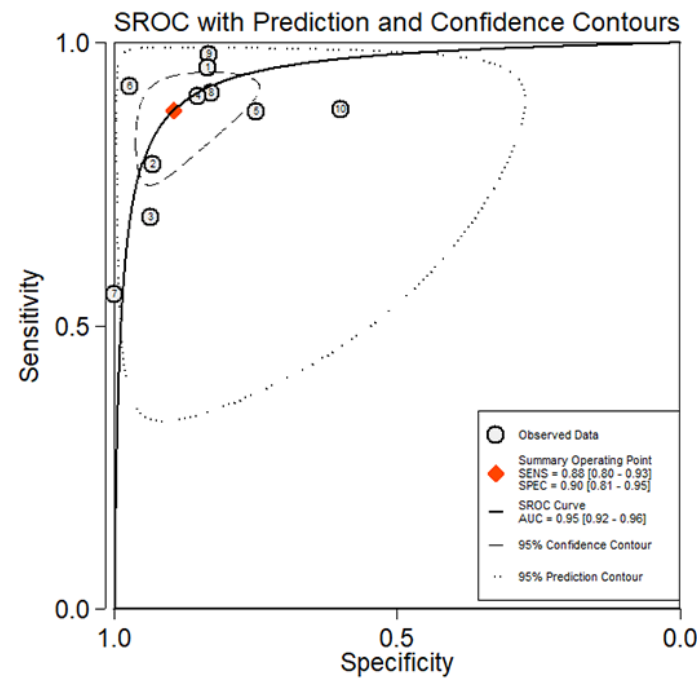

Figure 5. SROC. The confidence region (smaller circle with dash line) represents the ellipsoid $95 \%$ confidence region in SROC space for the summary point estimate of diagnostic performance. SROC, summary receiver operating characteristics curve; AUC, area under the curve; SPEC, Specificity; SENS, Sensitivity.

Sensitivity was significantly higher for studies not using hepatobiliary phase compared with those using hepatobiliary phase $(\mathrm{P}<0.001)$. Specificity was significantly higher for studies using a $3 \mathrm{~T}$ magnetic field compared with those using $1.5 \mathrm{~T}$ magnetic field $(\mathrm{P}=0.03)$. There were no significant differences in either the sensitivity or in specificity for the remaining study characteristics (all $\mathrm{P}>0.05$ ).

Additional value of DWI for contrast-enhanced MRI. The comparisons in the diagnostic performance of the different combinations of MRI protocols in the diagnosis of small HCC lesions are shown in Table IV. The sensitivity of DWI with contrast-enhanced MRI was significantly higher compared with that in contrast-enhanced MRI alone (0.89 vs. 0.78 ; $\mathrm{P}=0.01$ ). However, there was no significant difference for the specificity between DWI with contrast-enhanced MRI and conventional MRI alone $(\mathrm{P}=0.603)$.

\section{Discussion}

The aim of the present meta-analysis was to assess the diagnostic performance of DWI combined with conventional MRI for the diagnosis of small HCC lesions, with a diameter $\leq 2 \mathrm{~cm}$. The results suggested that DWI with conventional MRI had a high sensitivity of $88 \%$ and specificity of $90 \%$. The meta-regression analysis revealed that the heterogeneity of the pooled sensitivity may be partially attributed to whether hepatobiliary phase was used in the diagnosis of small HCC. In addition, the heterogeneity for the pooled specificity may be caused by the different magnetic fields used. However, a threshold effect was not identified.

Non-contrast enhanced ultrasonography (US) is a common choice for HCC screening in patients who with chronic liver 
Table III. Subgroup analysis and meta-regression.

\begin{tabular}{|c|c|c|c|c|c|c|}
\hline Characteristic & $\begin{array}{l}\text { No. of } \\
\text { studies }\end{array}$ & (Refs.) & $\begin{array}{c}\text { Pooled } \\
\text { sensitivity }(\mathrm{CI})\end{array}$ & P-value & $\begin{array}{c}\text { Pooled } \\
\text { specificity }(\mathrm{CI})\end{array}$ & P-value \\
\hline MRI field strength, $\mathrm{T}$ & & & & 0.76 & & 0.03 \\
\hline 1.5 & 6 & $(7,8,15,16,18,21)$ & $0.91(0.85-0.97)$ & & $0.85(0.75-0.95)$ & \\
\hline 3.0 & 4 & $(17,19,20,22)$ & $0.81(0.68-0.93)$ & & $0.94(0.87-1.00)$ & \\
\hline Country of origin & & & & 0.10 & & 0.85 \\
\hline Asia & 6 & $(8,17-19,20,22)$ & $0.87(0.78-0.95)$ & & $0.92(0.84-0.99)$ & \\
\hline Non-Asia & 4 & $(7,15,16,21)$ & $0.90(0.81-0.98)$ & & $0.86(0.74-0.98)$ & \\
\hline Study design & & & & 0.49 & & 0.34 \\
\hline Prospective & 3 & $(15,16,21)$ & $0.90(0.80-1.00)$ & & $0.88(0.75-1.00)$ & \\
\hline Retrospective & 7 & $(7,8,17-19,20,22)$ & $0.87(0.79-0.95)$ & & $0.90(0.82-0.98)$ & \\
\hline Hepatobiliary phase imaging & & & & $<0.001$ & & 0.92 \\
\hline Yes & 5 & $(16-19,22)$ & $0.81(0.72-0.91)$ & & $0.94(0.91-0.98)$ & \\
\hline No & 5 & $(7,8,15,20,21)$ & $0.93(0.88-0.98)$ & & $0.79(0.69-0.88)$ & \\
\hline
\end{tabular}

Table IV. Comparison of the diagnostic performance of DWI+ CE MRI with CE MRI alone.

\begin{tabular}{lcccr}
\hline Diagnostic methods & Pooled sensitivity (CI) & P-value & Pooled specificity (CI) & P-value \\
\hline DWI+ CE MRI & $0.89(0.83-0.94)$ & 0.01 & $0.88(0.78-0.94)$ & 0.603 \\
CE MRI & $0.78(0.74-0.83)$ & & $0.90(0.79-0.95)$ & \\
\hline
\end{tabular}

DWI, diffusion weighted imaging; CE, contrast-enhanced; MRI, magnetic resonance imaging.

disease, as it is cost-effective (23). However, there is low sensitivity when compared with that in contrast-enhanced computer tomography (CT) and MRI (24). Contrast-enhanced US has emerged as a promising method to diagnose small HCCs (25); however, additional studies are required to confirm its clinical value (23). Multiple meta-analyses have found that contrast-enhanced MRI outperforms contrast enhanced CT in the diagnosis of small HCCs with higher sensitivity and overall accuracy $(26,27)$. Previous meta-analysis indicated that contrast-enhanced MRI had moderately high sensitivity and high specificity in the diagnosis of small HCC (28). The present meta-analysis suggested that DWI combined with conventional MRI increased the sensitivity in the diagnosis of small HCC, whilst maintaining a high specificity. It is hypothesized that the ability to suppress the background signal of the liver parenchyma underlies the improved ability of DWI to detect smaller lesions $(29,30)$.

Li et al (31), found that DWI combined with gadoxetic acid disodium-enhanced MRI was beneficial to diagnose HCC and improved the specificity. However, the capability of contrast-enhanced MRI with DWI to diagnose small HCC lesions was not compared, which was investigated in the present meta-analysis. The present analysis found an increased sensitivity while maintaining high specificity using a combined method to diagnose small HCCs compared with using contrast-enhanced MRI alone. HCC lesions $<2 \mathrm{~cm}$ are less frequently presented during imaging compared with larger HCC lesions, including arterial enhancement, portal/equilibrium washout and T2 hyperintensity (32). The increase in sensitivity using the combined method could be due to the small HCCs presenting with hyperintensity in DWI (33).

ADC has been used to diagnose benign and malignant hepatic lesions (34). A previous study suggested that ADC was lower in malignant lesions, such as HCC and metastases, compared with that in benign lesions, such as cysts and hemangiomas (35). However, it is difficult to define a threshold of ADC value for benign and malignant liver lesions differentiation (36). An increasing number of studies have suggested that ADC is more accurate in grading smaller HCCs $(37,38)$, and for monitoring early treatment responses of HCC to radiofrequency ablation (39). In the 10 studies included in the present meta-analysis, only one study used a predetermined threshold ADC value to diagnose small HCC (20), and no difference was found in the ADC value between benign and malignant hepatic lesions. The remaining 9 studies used hyperintensity of the lesion compared with that in the liver background in the DWI, as one of the diagnostic criteria for HCC. The present analysis suggested that DWI may be used straightaway, in different diagnostic centers, without using a cut-off ADC value, which may differ between studies.

The value of DWI for the diagnosis of $\mathrm{HCC} \leq 1 \mathrm{~cm}$ requires further investigation as only 2 of the 10 studies compared the diagnostic performance of DWI for $\mathrm{HCC} \leq 1 \mathrm{~cm}(16,18)$. Both studies found that the sensitivity could be increased by adding DWI, which suggested the importance of using DWI in the diagnosis of HCCs with smaller lesions. 
There were several limitations in the present meta-analysis. Notable heterogeneity among the included studies was found, which may affect the applicability of the summery estimates. In addition, the majority of the included studies were retrospective studies (7 out of 10), in which confounding factors and bias are more common compared with that in prospective studies.

In conclusion, DWI in combination with conventional MRI is beneficial for the diagnosis of small HCC, which may increase the diagnostic sensitivity whilst maintaining high specificity.

\section{Acknowledgements}

The authors would like to thank Professor Xiaojia Zhang (Taiyuan Centers for Disease Control and Prevention, Taiyuan City, China) for her statistical assistance.

\section{Funding}

No funding was received.

\section{Availability of data and materials}

All data generated or analyzed during this study are included in this published article.

\section{Authors' contributions}

HL, GL and WZZ designed the study. HL, GL and WZZ acquired the data. GL drafted the initial manuscript. All authors revised and approved the final version of the manuscript.

\section{Ethics approval and consent to participate}

Not applicable.

\section{Patient consent for publication}

Not applicable.

\section{Competing interests}

The authors declare that they have no competing interests.

\section{References}

1. Wallace MC, Preen D, Jeffrey GP and Adams LA: The evolving epidemiology of hepatocellular carcinoma: A global perspective. Expert Rev Gastroenterol Hepatol 9: 765-779, 2015.

2. Hackl C, Schlitt HJ, Kirchner GI, Knoppke B and Loss M: Liver transplantation for malignancy: Current treatment strategies and future perspectives. World J Gastroenterol 20: 5331-5344, 2014.

3. Wald C, Russo MW, Heimbach JK, Hussain HK, Pomfret EA and Bruix J: New OPTN/UNOS policy for liver transplant allocation: Standardization of liver imaging, diagnosis, classification, and reporting of hepatocellular carcinoma. Radiology 266: 376-382, 2013.

4. Zamora-Valdes D, Taner T and Nagorney DM: Surgical treatment of hepatocellular carcinoma. Cancer Control 24 $1073274817729258,2017$.

5. Balogh J, Victor D III, Asham EH, Burroughs SG, Boktour M, Saharia A, Li X, Ghobrial RM and Monsour HP Jr: Hepatocellular carcinoma: A review. J Hepatocell Carcinoma 3: 41-53, 2016.
6. Forner A, Vilana R, Ayuso C, Bianchi L, Solé M, Ayuso JR, Boix L, Sala M, Varela M, Llovet JM, et al: Diagnosis of hepatic nodules $20 \mathrm{~mm}$ or smaller in cirrhosis: Prospective validation of the noninvasive diagnostic criteria for hepatocellular carcinoma. Hepatology 47: 97-104, 2008.

7. Le Moigne F, Durieux M, Bancel B, Boublay N, Boussel L, Ducerf C, Berthezène Y and Rode A: Impact of diffusion-weighted MR imaging on the characterization of small hepatocellular carcinoma in the cirrhotic liver. Magn Reson Imaging 30: 656-665, 2012.

8. Xu PJ, Yan FH, Wang JH, Lin J and Ji Y: Added value of breathhold diffusion-weighted MRI in detection of small hepatocellular carcinoma lesions compared with dynamic contrast-enhanced MRI alone using receiver operating characteristic curve analysis. J Magn Reson Imaging 29: 341-349, 2009.

9. Kim SS, Kim SH, Song KD, Choi SY and Heo NH: Value of gadoxetic acid-enhanced MRI and diffusion-weighted imaging in the differentiation of hypervascular hyperplastic nodule from small $(<3 \mathrm{~cm})$ hypervascular hepatocellular carcinoma in patients with alcoholic liver cirrhosis: A retrospective case-control study. J Magn Reson Imaging 51: 70-80, 2020.

10. Zhong X, Tang H, Lu B, You J, Piao J, Yang P and Li J: Differentiation of small hepatocellular carcinoma from dysplastic nodules in cirrhotic liver: Texture analysis based on MRI improved performance in comparison over gadoxetic acid-enhanced MR and diffusion-weighted imaging. Front Oncol 9: 1382, 2020.

11. Whiting PF, Rutjes AW, Westwood ME, Mallett S, Deeks JJ, Reitsma JB, Leeflang MM, Sterne JA and Bossuyt PM; QUADAS-2 Group: QUADAS-2: A revised tool for the quality assessment of diagnostic accuracy studies. Ann Intern Med 155: 529-536, 2011.

12. Reitsma JB, Glas AS, Rutjes AW, Scholten RJ, Bossuyt PM and Zwinderman AH: Bivariate analysis of sensitivity and specificity produces informative summary measures in diagnostic reviews. J Clin Epidemiol 58: 982-990, 2005.

13. Choo SP, Tan WL, Goh BKP, Tai WM and Zhu AX: Comparison of hepatocellular carcinoma in Eastern versus Western populations. Cancer 122: 3430-3446, 2016.

14. Basha MAA, Refaat R, Mohammad FF, Khamis MEM, El-Maghraby AM, El Sammak AA, Al-Molla RM, Mohamed HAE, Alnaggar AA, Hassan HA, et al: The utility of diffusion-weighted imaging in improving the sensitivity of LI-RADS classification of small hepatic observations suspected of malignancy. Abdom Radiol (NY) 44: 1773-1784, 2019.

15. Di Martino M, Di Miscio R, De Filippis G, Lombardo CV, Saba L, Geiger D and Catalano C: Detection of small $(\leq 2 \mathrm{~cm})$ $\mathrm{HCC}$ in cirrhotic patients: Added value of diffusion MR-imaging. Abdom Imaging 38: 1254-1262, 2013.

16. Hwang J, Kim YK, Kim JM, Lee WJ, Choi D and Hong SS: Pretransplant diagnosis of hepatocellular carcinoma by gadoxetic acid-enhanced and diffusion-weighted magnetic resonance imaging. Liver Transpl 20: 1436-1446, 2014.

17. Kwon HJ, Byun JH, Kim JY, Hong GS, Won HJ, Shin YM and Kim PN: Differentiation of small $(\leq 2 \mathrm{~cm})$ hepatocellular carcinomas from small benign nodules in cirrhotic liver on gadoxetic acid-enhanced and diffusion-weighted magnetic resonance images. Abdom Imaging 40: 64-75, 2015.

18. Park MJ,Kim YK, Lee MW, Lee WJ, Kim YS, Kim SH, Choi D and Rhim H: Small hepatocellular carcinomas: Improved sensitivity by combining gadoxetic acid-enhanced and diffusion-weighted MR imaging patterns. Radiology 264: 761-770, 2012.

19. Zhao XT, Li WX, Chai WM and Chen KM: Detection of small hepatocellular carcinoma using gadoxetic acid-enhanced MRI: Is the addition of diffusion-weighted MRI at 3.0T beneficial? J Dig Dis 15: 137-145, 2014.

20. Vandecaveye V, De Keyzer F, Verslype C, Op de Beeck K, Komuta M, Topal B, Roebben I, Bielen D, Roskams T, Nevens F and Dymarkowski S: Diffusion-weighted MRI provides additional value to conventional dynamic contrast-enhanced MRI for detection of hepatocellular carcinoma. Eur Radiol 19: 2456-2466, 2009.

21. Rhee H, Kim MJ, Park MS and Kim KA: Differentiation of early hepatocellular carcinoma from benign hepatocellular nodules on gadoxetic acid-enhanced MRI. Br J Radiol 85: e837-e844, 2012.

22. Tang A, Bashir MR, Corwin MT, Cruite I, Dietrich CF, Do RKG, Ehman EC, Fowler KJ, Hussain HK, Jha RC, et al: Evidence supporting LI-RADS major features for CT- and MR imaging-based diagnosis of hepatocellular carcinoma: A systematic review. Radiology 286: 29-48, 2018. 
23. Ronot M, Purcell Y and Vilgrain V: Hepatocellular carcinoma: Current imaging modalities for diagnosis and prognosis. Dig Dis Sci 64: 934-950, 2019.

24. Hanna RF, Miloushev VZ, Tang A, Finklestone LA, Brejt SZ, Sandhu RS, Santillan CS, Wolfson T, Gamst A and Sirlin CB: Comparative 13-year meta-analysis of the sensitivity and positive predictive value of ultrasound, CT, and MRI for detecting hepatocellular carcinoma. Abdom Radiol (NY) 41: 71-90, 2016.

25. Hsiao CY, Chen PD and Huang KW: A prospective assessment of the diagnostic value of contrast-enhanced ultrasound, dynamic computed tomography and magnetic resonance imaging for patients with small liver tumors. J Clin Med 8: 1353, 2019.

26. Liu X, Jiang H, Chen J, Zhou Y, Huang Z and Song B: Gadoxetic acid disodium-enhanced magnetic resonance imaging outperformed multidetector computed tomography in diagnosing small hepatocellular carcinoma: A meta-analysis. Liver Transpl 23: 1505-1518, 2017

27. Li J, Li X, Weng J, Lei L, Gong J, Wang J, Li Z, Zhang L and He S: Gd-EOB-DTPA dynamic contrast-enhanced magnetic resonance imaging is more effective than enhanced 64-slice CT for the detection of small lesions in patients with hepatocellular carcinoma. Medicine (Baltimore) 97: e13964, 2018.

28. Kierans AS, Kang SK and Rosenkrantz AB: The diagnostic performance of dynamic contrast-enhanced MR imaging for detection of small hepatocellular carcinoma measuring up to $2 \mathrm{~cm}$ : A meta-analysis. Radiology 278: 82-94, 2016.

29. Wu LM, Hu J, Gu HY, Hua J and Xu JR: Can diffusion-weighted magnetic resonance imaging (DW-MRI) alone be used as a reliable sequence for the preoperative detection and characterisation of hepatic metastases? A meta-analysis. Eur J Cancer 49: 572-584, 2013

30. Mannelli L, Bhargava P, Osman SF, Raz E, Moshiri M, Laffi G, Wilson GJ and Maki JH: Diffusion-weighted imaging of the liver: A comprehensive review. Curr Probl Diagn Radiol 42: 77-83, 2013.

31. Li X, Li C, Wang R, Ren J, Yang J and Zhang Y: Combined application of gadoxetic acid disodium-enhanced magnetic resonance imaging (MRI) and diffusion-weighted imaging (DWI) in the diagnosis of chronic liver disease-induced hepatocellular carcinoma: A meta-analysis. PLoS One 10: e0144247, 2015.

32. van den Bos IC, Hussain SM, Dwarkasing RS, Hop WC, Zondervan PE, de Man RA, IJzermans JN, Walker CW and Krestin GP: MR imaging of hepatocellular carcinoma: Relationship between lesion size and imaging findings, including signal intensity and dynamic enhancement patterns. J Magn Reson Imaging 26: 1548-1555, 2007.
33. Parikh T, Drew SJ, Lee VS, Wong S, Hecht EM, Babb JS and Taouli B: Focal liver lesion detection and characterization with diffusion-weighted MR imaging: Comparison with standard breath-hold T2-weighted imaging. Radiology 246: 812-822, 2008.

34. Pankaj Jain T, Kan WT, Edward S, Fernon H and Kansan Naider R: Evaluation of $\mathrm{ADC}_{\text {ratio }}$ on liver MRI diffusion to discriminate benign versus malignant solid liver lesions. Eur J Radiol Open 5: 209-214, 2018.

35. Goshima S, Kanematsu M, Kondo H, Yokoyama R, Kajita K, Tsuge Y, Watanabe H, Shiratori Y, Onozuka M and Moriyama N: Diffusion-weighted imaging of the liver: Optimizing $b$ value for the detection and characterization of benign and malignant hepatic lesions. J Magn Reson Imaging 28: 691-697, 2008

36. Miller FH, Hammond N, Siddiqi AJ, Shroff S, Khatri G, Wang Y, Merrick LB and Nikolaidis P: Utility of diffusion-weighted MRI in distinguishing benign and malignant hepatic lesions. J Magn Reson Imaging 32: 138-147, 2010.

37. Le Moigne F, Boussel L, Haquin A, Bancel B, Ducerf C, Berthezène $\mathrm{Y}$ and Rode $\mathrm{A}$ : Grading of small hepatocellular carcinomas $(\leq 2 \mathrm{~cm})$ : Correlation between histology, T2 and diffusion-weighted imaging. Br J Radiol 87: 20130763 , 2014.

38. Okamura S, Sumie S, Tonan T, Nakano M, Satani M, Shimose S, Shirono T, Iwamoto $\mathrm{H}$, Aino H, Niizeki T, et al: Diffusion-weighted magnetic resonance imaging predicts malignant potential in small hepatocellular carcinoma. Dig Liver Dis 48: 945-952, 2016.

39. Mori Y, Tamai H, Shingaki N, Moribata K, Deguchi H, Ueda K, Inoue I, Maekita T, Iguchi M, Kato J, et al: Signal intensity of small hepatocellular carcinoma on apparent diffusion coefficient mapping and outcome after radiofrequency ablation. Hepatol Res 45: 75-87, 2015.

This work is licensed under a Creative Commons Attribution-NonCommercial-NoDerivatives 4.0 International (CC BY-NC-ND 4.0) License. 